\title{
A Low-Dimensional Model for the Maximal Amplification Factor of Bichromatic Wave Groups
}

\author{
W. N. $\operatorname{Tan}^{1, *} \&$ Andonowati ${ }^{2}$ \\ ${ }^{1}$ Fakulti Sains, Universiti Teknologi Malaysia \\ ${ }^{2}$ Department of Mathematics, Institut Teknologi Bandung, Indonesia
}

\begin{abstract}
We consider a low-dimensional model derived from the nonlinearSchrödinger equation that describes the evolution of a special class of surface gravity wave groups, namely bichromatic waves. The model takes only two modes into account, namely the primary mode and the third order mode which is known to be most relevant for bichromatic waves with small frequency difference. Given an initial condition, an analytical expression for the maximal amplitude of the evolution of this initial wave group according to the model can be readily obtained. The aim of this investigation is to predict the amplification factor defined as the quotient between the maximal amplitude over all time \& space and the initial maximal amplitude. Although this is a problem of general interest, as a case study, initial conditions in the form of a bichromatic wave group are taken. Using the low dimensional model it is found that the least upper bound of the maximal amplification factor for this bichromatic wave group is $\sqrt{2}$. To validate the analytical results of this model, a numerical simulation on the full model is also performed. As can be expected, good agreement is observed between analytical and numerical solutions for a certain range of parameters; when the initial amplitude is not too large, or when the difference of frequency is not too small. The results are relevant and motivated for the generation of waves in hydrodynamic laboratories.
\end{abstract}

Keywords: amplification factor; amplitude; bichromatic; frequency difference; lowdimensional model; non-linear Schrödinger equation.

\section{$1 \quad$ Introduction}

The motivation of this problem stems from the study of generating extreme waves in towing tanks of hydrodynamic laboratories. These tanks are used to test the performance of the maritime structures on scaled models, so that designers can collect the hydrodynamic properties of their designs before the actual construction of such structures. For safety reasons, requirements for these constructions are becoming more stringent. In many cases, it is necessary that the scaled models are tested under extreme conditions, requiring that waves with large amplitudes to be generated at least at the position of the tested

\footnotetext{
* Permanent staff, Faculty of Engineering, Multimedia University, Malaysia
} 
models in the tanks. Thus a study of deterministic extreme wave generation is becoming more and more relevant. The physical limitations of the wave makers as well as the non-linear evolution of the water make the generation of extreme wave non-trivial. Influences of nonlinearity, for example, result into deformation of signals that are produced at the wave makers while propagating away along the tank. It is therefore interesting to investigate how these signals deform and how large their amplitude can increase from the generated signal along the tank.

Influences of nonlinearity in the wave groups evolutions have been observed by several authors. In bichromatic wave groups, large deformations and increase of amplitude can be found during long-time evolutions. Lo \& Mei (1985) reported that the long-run of a bichromatic wave consists of the splitting of the original group into two groups [1]. A comparable experimental result was provided by Stansberg (1997). The measured wave groups in a wave tank that were generated using only two frequencies, showed large deviations from the linear theory after some distance from the wave maker [2]. This was also clearly demonstrated numerically by Westhuis (2001), in which the evolution of the bichromatic waves shows the splitting of the original envelope into two distinguishable envelopes [3]. Somewhat more theoretical investigation of the bichromatic wave deformation is described in [4], [5] where the frequency difference between the two monochromatics play importance role in the deformation of waves that are initially Bichromatic. Many investigations on wave deformation are reported in work related to the stability of another form of nonlinear wave groups, namely a slightly modulated uniform wave train. Among the first few who worked on these are Lighthill (1965) [6], Benjamin \& Feir (1967) [7]. Related to modulated wave trains, a number of papers such as in [8], [9] and [10] showed that the maximal amplification factor due to Benjamin $\&$ Feir instability is three.

In this paper, instead of looking at a signaling problem as described above, we will consider the initial value problem. Here, an initial profile is given and the evolution of this profile will be investigated. It is known that the superposition of two waves of amplitudes $a_{1}$ and $a_{2}$ in a linear medium produces a wave with a maximal amplitude of $\left(a_{1}+a_{2}\right)$. But in realistic evolution of waves, the presence of non linearity can dominate the deformation of wave evolution and this maximal amplitude will be different, say $m\left(a_{1}+a_{2}\right)$ where $m$ is an amplification factor due to nonlinear effects.

The aim of this investigation is to find the maximal amplification factor, $m$ for the evolution of waves that are initially of the forms of bichromatic wave groups. In a realistic investigation, the full three dimensional effects as well as 
the full equations describing the surface waves have to be taken into account, but they are generally too difficult to study. As an initial investigation, we construct a low-dimensional model for the focusing nonlinear-Schrödinger (NLS) equation, in which these effects are incorporated in a simplified way. To assess the validity of this truncated model, we perform a numerical simulation on the full model of NLS equation starting from the initial conditions consistent with the one used in the low-dimensional model. The numerical scheme is based on the discretization of the variational structure for space variable, with an implicit Crank-Nicolson scheme for time variable.

The organization of this paper is as follows. In section 2, we present the mathematical model used in describing the surface waves. In section 3, a lowdimensional model will be derived to describe the evolutions of bichromatic wave groups. Section 4 discusses the analytical results on maximal amplification factor based on the low-dimensional model. Comparisons of analytical results with numerical simulations are given in section 5. Conclusions and remarks complete the paper.

\section{Problem Description}

A full description of ideal surface gravity waves consists of an equation for the interior fluid domain together with boundary conditions on the water surface as well as on the bottom. Assuming that the waves propagate in one direction and are not 'too long' and not 'too large', the interior properties of the fluid can be transformed into the surface to form an asymptotic model for the surface gravity waves. One of such asymptotic models for the surface elevation $\eta(x, t)$ is known as a (modified) Korteweg-de Vries equation

$$
\frac{\partial \eta}{\partial t}+i \Omega\left(-i \frac{\partial}{\partial x}\right) \eta=-\frac{3}{2} \eta \frac{\partial \eta}{\partial x}
$$

where $\Omega(k)=k \sqrt{\tanh (k) / k}$ is the frequency and $k$ is the wave number [11]. The variables here are normalized in dimensionless coordinates. These scaled variables are related to physical laboratory coordinates $\eta_{l a b}, x_{l a b}, t_{l a b}, \Omega_{l a b}$, and $k_{\text {lab }}$ by the following transformations:

$$
\eta_{l a b}=h \eta, \quad x_{l a b}=h x, \quad t_{l a b}=\sqrt{\frac{h}{g}} t, \quad \Omega_{l a b}=\sqrt{\frac{g}{h}} \Omega, \quad k_{l a b}=\frac{k}{h},
$$

with $h$ the depth of the undisturbed water in the tank and $g$ the acceleration of gravity. 
Looking for a solution in the form of a slow deformation of a harmonic mode of a central wave number $k_{0}$ and frequency $\omega_{0}$ related by the dispersion relation $\omega_{0}=\Omega\left(k_{0}\right)=\sqrt{k_{0} \tanh \left(k_{0}\right)}$, the surface elevation can be written in the form

$$
\eta(x, t)=A(\xi, \tau) e^{i\left(k_{0} x-\omega_{0} t\right)}+c . c .
$$

with a complex slowly varying envelope $A(\xi, \tau)$ where variables in a coordinate system moving with the group velocity $\Omega^{\prime}\left(k_{0}\right)$ are introduced: $\xi=\varepsilon\left(x-\Omega^{\prime}\left(k_{0}\right) t\right)$ and $\tau=\varepsilon^{2} t$. Then the complex amplitude satisfies the NLS equation

$$
\frac{\partial A}{\partial \tau}+i \beta \frac{\partial^{2} A}{\partial \xi^{2}}+i \gamma|A|^{2} A=0
$$

where the parameters $\beta$ and $\gamma$ are given by [11] $\beta=-\frac{1}{2} \Omega^{\prime \prime}\left(k_{0}\right)$ $\gamma=\frac{9}{4} k_{0}\left(\sigma_{0}+\sigma_{2}\right), \sigma_{0}=1 / \Omega^{\prime}\left(k_{0}\right)-\Omega^{\prime}(0)$, and $\sigma_{2}=k_{0} /\left(2 \Omega\left(k_{0}\right)-\Omega\left(2 k_{0}\right)\right)$. The slow variables $\xi, \tau$ are related to the physical variables as follows:

$$
x_{l a b}=\frac{h}{\varepsilon}\left(\xi+\frac{\Omega^{\prime}\left(k_{0}\right)}{\varepsilon} \tau\right), t_{l a b}=\frac{1}{\varepsilon^{2}} \sqrt{\frac{h}{g}} \tau .
$$

The NLS equation has infinitely many conserved quantities, among them are

$$
\begin{aligned}
& N(A)=\int|A|^{2} d \xi, \\
& L(A)=\operatorname{Im} \int \bar{A} \partial_{\xi} A d \xi .
\end{aligned}
$$

The first integral can be interpreted as the wave energy, and its flow expresses the Gauge invariance of NLS equation. The second one is known as linear momentum since its flow is translation symmetry.

It is well known that wave groups, satisfying NLS, have essentially different behavior depending on whether $\beta$ and $\gamma$ have equal sign or not [8]:

- If $\beta$ and $\gamma$ have the same sign, i.e. $\beta \gamma>0$, then (3) is known as the focusing NLS equation.

- If $\beta$ and $\gamma$ have different sign, i.e. $\beta \gamma<0$, then (3) is known as the defocusing NLS equation.

The wave number $k_{c r i t}$ for which $\beta \gamma=0$ holds, is called the critical wave number. In our study, we consider the focusing NLS equation and we will 
concentrate on investigating evolution of the envelope of bichromatic waves. A bichromatic wave group is a linear superposition of two regular waves with different frequencies. Specifically, if we choose the two monochromatic components to have equal amplitude, say $q$, and frequencies $\omega_{1}$ and $\omega_{2}$ then the resulting bichromatic wave can be written in the form

$$
\begin{aligned}
\eta(x, t) & =q \cos \left(k_{1} x-\omega_{1} t\right)+q \cos \left(k_{2} x-\omega_{2} t\right) \\
& =q \cos (\bar{\kappa} x-\bar{v} t) e^{i\left(k_{0} x-\omega_{0} t\right)}+c . c .,
\end{aligned}
$$

with $\bar{\kappa}=\frac{1}{2}\left(k_{1}-k_{2}\right), k_{0}=\frac{1}{2}\left(k_{1}+k_{2}\right), \bar{v}=\frac{1}{2}\left(\omega_{1}-\omega_{2}\right)$ and $\omega_{0}=\frac{1}{2}\left(\omega_{1}+\omega_{2}\right)$. The central wave number $k_{0}$ and frequency $\omega_{0}$ are related by the dispersion $\omega_{0}=\Omega\left(k_{0}\right)=k_{0} \sqrt{\tanh \left(k_{0}\right) / k_{0}}$. The expression for the elevation $\eta(x, t)$ can be interpreted as a harmonic carrier wave $e^{i\left(k_{0} x-\omega_{0} t\right)}$ modulated by a slow varying amplitude $\hat{A}(x, t)=q \cos (\bar{\kappa} x-\bar{v} t)$. In terms of slow variables, this complex amplitude can be written as

$$
A(\xi, \tau)=a_{0} \cos (\kappa \xi-v \tau),
$$

with $\mathrm{a}_{0}=\frac{q}{\varepsilon}, \kappa=\frac{\bar{\kappa}}{\varepsilon}$, and $v=\frac{1}{\varepsilon^{2}}\left(\bar{\kappa} \Omega^{\prime}\left(k_{0}\right)-\bar{v}\right)$.

\section{Low-Dimensional Model}

In view of (8), we concentrate on the bichromatic wave group with the initial condition:

$$
A(\xi, \tau=0)=a_{0} \cos \kappa \xi .
$$

We approximate the solution $A(\xi, \tau)$ simply by a first order mode induced from the initial condition, and a third order mode generated from the cubic nonlinearity. Thus, the Ansatz of this low-dimensional model takes the form of

$$
A(\xi, \tau)=a(\tau) \cos (\kappa \xi)+b(\tau) \cos (3 \kappa \xi),
$$

where $a(\tau), b(\tau) \in \mathbb{C}$. In other words, we study the projections of the full solutions $A(\xi, \tau)$ to the two base functions, $\cos (\kappa \xi)$ and $\cos (3 \kappa \xi)$. Observe that the periodicity of $\xi$ in the solutions remain $\frac{2 \pi}{\kappa}$ for $\tau \geq 0$. Hence we restrict our interests in investigating the equation on an interval length of one period $\frac{2 \pi}{\kappa}$ in $\xi$ with periodic boundary conditions. 
In order to obtain the dynamic equations of $a(\tau)$ and $b(\tau)$, we consider the NLS equation as a Hamiltonian system. The NLS equation is the EulerLagrange equation from the action principle with the action functional

$$
\mathcal{L}(A)=\int d \tau \int i \bar{A} \frac{\partial A}{\partial \tau} d \xi-\mathcal{H}(A),
$$

where the Hamiltonian function is

$$
\mathcal{H}(A)=\frac{1}{2} \int \beta\left|\frac{\partial A}{\partial \xi}\right|^{2}-\gamma|A|^{4} d \xi .
$$

We restrict the functional $\mathcal{L}(A)$ to amplitudes $A$ in the manifold $\mathcal{M}$ :

$$
\mathcal{M}=\{A(\xi, \tau)=a(\tau) \cos (\kappa \xi)+b(\tau) \cos (3 \kappa \xi) \mid a(\tau), b(\tau) \in \mathbb{C}\} .
$$

Upon elaborating, the resulting functional is

$$
\begin{aligned}
\mathcal{L}^{\star}(a, b)= & \int \frac{i}{2}[\bar{a} \dot{a}+\bar{b} \dot{b}]-\frac{\beta}{2}\left[\kappa^{2}|a|^{2}+9 \kappa^{2}|b|^{2}\right] \\
& +\frac{\gamma}{4}\left[\frac{3}{4}|a|^{4}+\frac{|a|^{2}}{2}(\bar{a} b+a \bar{b})\right. \\
& \left.+(|a \| b|)^{2}+\frac{1}{2}(\bar{a} b+a \bar{b})^{2}+\frac{3}{4}|b|^{4}\right] d \tau .
\end{aligned}
$$

Equivalently, we could express the complex-valued coefficients $a(\tau)$ and $b(\tau)$ more compactly in exponential form as $a(\tau)=r_{1}(\tau) e^{i \theta_{1}(\tau)}$ and $b(\tau)=r_{2}(\tau) e^{i \theta_{2}(\tau)}$. The initial conditions under consideration give $r_{1}(0)=a_{0}$, $\mathrm{r}_{2}(0)=0$ and $\varphi(0)=0$, where $\varphi(\tau)=\theta_{1}(\tau)-\theta_{2}(\tau)$. With the above representations and by applying the initial condition, the conserved quantity (5) gives

$$
|a|^{2}+|b|^{2}=r_{1}^{2}+r_{2}^{2}=a_{0}^{2} .
$$

Therefore, the functional $\mathcal{L}^{\star}(a, b)$ can be written in terms of $r_{1}(\tau)$ and $\varphi(\tau)$ only. From the action principle, the Euler-Lagrange equations for $\mathcal{L}^{\star}(a, b)$ give the dynamics equations:

$$
\begin{aligned}
& \dot{\varphi}=8 \beta \kappa^{2}-\frac{\gamma}{4}\left[2 r_{1}^{2}-a_{0}^{2}+\frac{4 r_{1}^{3}-3 r_{1} a_{0}^{2}}{\sqrt{a_{0}^{2}-r_{1}^{2}}} \cos (\varphi)+2 \cos (2 \varphi)\left(2 r_{1}^{2}-a_{0}^{2}\right),\right. \\
& \dot{r}_{1}=\frac{\gamma}{4}\left[r_{1}^{2} \sqrt{a_{0}^{2}-r_{1}^{2}} \sin (\varphi)+2 r_{1}\left(a_{0}^{2}-r_{1}^{2}\right) \sin (2 \varphi)\right] .
\end{aligned}
$$


We note that similar dynamic equations for $a(\tau)$ and $b(\tau)$ or $r_{1}(\tau)$ and $\varphi(\tau)$ could also be obtained by projecting the NLS equation in the two basic directions or substituting (10) into NLS and truncating higher order terms. Further we observe that the dynamic differential equations (15) are in fact a Hamiltonian dynamical system, in which the Hamiltonian $\mathcal{H}^{*}$ for the lowdimensional model is given by

$$
\begin{aligned}
\mathcal{H}^{*}\left(r_{1}, \varphi\right)= & \frac{\beta \kappa^{2}}{2}\left(9 a_{0}^{2}-8 r_{1}^{2}\right)-\frac{\gamma}{4}\left[\frac{-1}{2} r_{1}^{4}+\frac{1}{2} r_{1}^{2} r_{0}^{2}+\frac{3}{4} a_{0}^{4}\right. \\
& \left.+r_{1}^{3} \sqrt{a_{0}^{2}-r_{1}^{2}} \cos \varphi+r_{1}^{2}\left(a_{0}^{2}-r_{1}^{2}\right) \cos (2 \varphi)\right] .
\end{aligned}
$$

Since the dynamic differential equations (15) are autonomous, the Hamiltonian system $\mathcal{H}^{*}\left(r_{1}, \varphi\right)$ is conservative. Thus it is constant on solutions. By applying the initial condition,

$$
\mathcal{H}^{*}\left(a_{0}, 0\right)=\frac{\beta \kappa^{2} a_{0}^{2}}{2}-\frac{3 \gamma}{16} a_{0}^{4},
$$

we have the Hamiltonian equation as follows:

$$
\begin{aligned}
4 \beta \kappa^{2}\left(a_{0}^{2}-r_{1}^{2}\right) & -\frac{\gamma}{4}\left[\frac{-1}{2} r_{1}^{4}+\frac{1}{2} r_{1}^{2} r_{0}^{2}\right. \\
& \left.+r_{1}^{3} \sqrt{a_{0}^{2}-r_{1}^{2}} \cos \varphi+r_{1}^{2}\left(a_{0}^{2}-r_{1}^{2}\right) \cos (2 \varphi)\right]=0 .
\end{aligned}
$$

The domain for $r_{1}$ is $0 \leq r_{1} \leq a_{0}$, as a result from the conservation property. Substituting $\cos (2 \varphi)=2 \cos ^{2} \varphi-1$ to the above equation, we have a quadratic equation with the roots are given by

$$
|\cos \varphi|=\left|\frac{\frac{\gamma}{4} r_{1}^{2} \pm \sqrt{\frac{\gamma}{4}\left[\frac{\gamma}{4} r_{1}^{4}+\left(a_{0}^{2}-r_{1}^{2}\right)\left(32 \beta \kappa^{2}+\gamma r_{1}^{2}\right]\right.}}{-\gamma r_{1} \sqrt{a_{0}^{2}-r_{1}^{2}}}\right| \leq 1,
$$

where the inequality resulted from the condition that $|\cos \varphi| \leq 1$.

\section{$4 \quad$ Analytical Results}

Our objective is to look for the maximal amplification factor defined by:

$$
\alpha=\frac{\max _{\tau, \xi}|A(\xi, \tau)|}{\max _{\xi}|A(\xi, 0)|} .
$$


Note that choice of $A(\xi, \tau)$ in (10) gives

$$
|A|^{2}=r_{1}^{2} \cos ^{2}(\kappa \xi)+r_{2}^{2} \cos ^{2}(3 \kappa \xi)+2 r_{1} r_{2} \cos ^{2}(\kappa \xi)\left(4 \cos ^{2}(\kappa \xi)-3\right) \cos \varphi .
$$

The value of $\cos \varphi$ from (17) can take positive or negative value. However, the level curves of the Hamiltonian system always give positive values for $\cos \varphi$ so that $\max _{\xi \tau}|A(\xi, \tau)|$ occurs when the each term in the expression for $|A|^{2}$ above maximum, namely at $\xi=n \pi / \kappa, n \in \mathbb{Z}$. Thus, for each $t_{\text {lab }}$, the maximal amplitude of wave groups evolutions always occur at the laboratory position given by

$$
x_{l_{a b}}=\frac{h}{\varepsilon}\left(\frac{n \pi}{\kappa}+\Omega^{\prime} \varepsilon \sqrt{\frac{g}{h}} t_{l_{a b}}\right), \quad n \in \mathbb{Z} .
$$

For simplification, we take $n=0$, and restrict our interest on $\max _{\tau}|A(0, \tau)|$. By the conservation property from (14), we have a two-dimensional optimization problem as:

$$
\alpha=\frac{\max _{\tau, \xi}|A(\xi, \tau)|}{\max _{\xi}|A(\xi, 0)|}=\frac{\max _{\tau} \sqrt{a_{0}^{2}+2 r_{1} \sqrt{\left(a_{0}^{2}-r_{1}^{2}\right)} \cos \varphi}}{a_{0}} .
$$

Without considering the restriction from the Hamiltonian system, the upper bound of the maximal amplification factor is $\sqrt{2}$.

Basically we could just investigate $\max _{\tau}\left\{2 r_{1} \sqrt{\left(a_{0}^{2}-r_{1}^{2}\right)} \cos \varphi\right\}$, where $r_{1}(\tau)$ and $\varphi(\tau)$ are governed by the dynamic equations (15). This gives us:

$$
\max _{\tau}\left\{2 r_{1} \sqrt{\left(a_{0}^{2}-r_{1}^{2}\right)} \cos \varphi\right\}
$$

subject to:

$$
\begin{aligned}
& 4 \beta \kappa^{2}\left(a_{0}^{2}-r_{1}^{2}\right)-\frac{\gamma}{4}\left[\frac{-1}{2} r_{1}^{4}+\frac{1}{2} r_{1}^{2} r_{0}^{2}\right. \\
& \left.+r_{1}^{3} \sqrt{a_{0}^{2}-r_{1}^{2}} \cos \varphi+r_{1}^{2}\left(a_{0}^{2}-r_{1}^{2}\right) \cos (2 \varphi)\right]=0
\end{aligned}
$$

where $0 \leq \mathrm{r}_{1} \leq \mathrm{a}_{0}$. 
Substitution of the expression for $\cos \varphi$ in (17) to the objective function above, we are able to reduce the problem to a one-dimensional optimization problem. Instead of using time variable as the parameter of optimization, we can use $r_{1}$ giving

$$
\max _{r_{1}}\left\{\frac{-2}{\gamma}\left[\frac{\gamma}{4} r_{1}^{2}+\sqrt{\frac{\gamma}{4}\left[\frac{\gamma}{4} r_{1}^{4}+\left(a_{0}^{2}-r_{1}^{2}\right)\left(32 \beta \kappa^{2}+\gamma r_{1}^{2}\right)\right]}\right]\right\}
$$

where $r_{1}$ satisfies

$$
\frac{\frac{\gamma}{4} r_{1}^{2}+\sqrt{\frac{\gamma}{4}\left[\frac{\gamma}{4} r_{1}^{4}+\left(a_{0}^{2}-r_{1}^{2}\right)\left(32 \beta \kappa^{2}+\gamma r_{1}^{2}\right)\right]}}{-\gamma r_{1} \sqrt{a_{0}^{2}-r_{1}^{2}}} \leq 1 \text {, and } 0 \leq \mathrm{r}_{1} \leq \mathrm{a}_{0}, r_{1} \in \mathbb{R} .
$$

The corresponding critical points of (22) are given by

$$
\frac{-\sqrt{3}}{3 \gamma} \sqrt{2 \gamma^{2} a_{0}^{2}-64 \gamma \beta \kappa^{2} \pm \sqrt{\gamma^{4} a_{0}^{4}+32 \gamma^{3} a_{0}^{2} \beta \kappa^{2}+1024 \kappa^{4} \beta^{2} \gamma^{2}}} .
$$

Thus, the solutions to this one-dimensional optimization problem (22) are the critical points (24) or the boundary points governed the domain defined in (23). Examining these solutions, we obtain the least upper bound of the maximal amplification factor $\sqrt{2}$.

\section{Graphical Representations and Comparisons}

We present here results in laboratory coordinates for the case of the wave tank with depth $h=5 \mathrm{~m}$. The wave tank taken here is with reference to the wave tank at MARIN (Maritime Research Institute Netherlands, Wageningen). By taking the acceleration of gravity $g=9.8 \mathrm{~m} / \mathrm{s}^{2}$, the critical wave number is $\mathrm{k}_{\text {crit }_{\text {ab }}}=0.229297$ in terms of laboratory coordinates, or $\mathrm{k}_{\text {crit }}=1.146037$ in terms of scaled coordinates. In our calculations, we must set the wave number $\mathrm{k}_{0_{\text {lab }}}>\mathrm{k}_{\text {crit }_{\text {ab }}}=0.229297$ to ensure that the NLS equation is of focusing type.

In this section, we are going to assess the analytical results obtained from the low-dimensional model. We first fix the frequencies and vary the initial amplitude. The values of the frequencies as well as the central wave number used in terms of laboratory coordinates are $\omega_{1_{\mathrm{lab}}}=3.30, \omega_{2_{\mathrm{lab}}}=2.99$, $\mathrm{k}_{0_{\mathrm{lab}}}=1.0119$. Alternatively, we fix the initial amplitude and vary the difference of the frequency. For this case, the difference of frequencies is given 
by $\bar{v}_{\text {lab }}=\frac{\omega_{1_{\text {lab }}}-\omega_{2_{\text {lab }}}}{2}$ for a fix initial amplitude $\mathrm{q}_{\text {lab }}=0.08$, with a fix $\omega_{1_{\mathrm{abb}}}=3.30$.

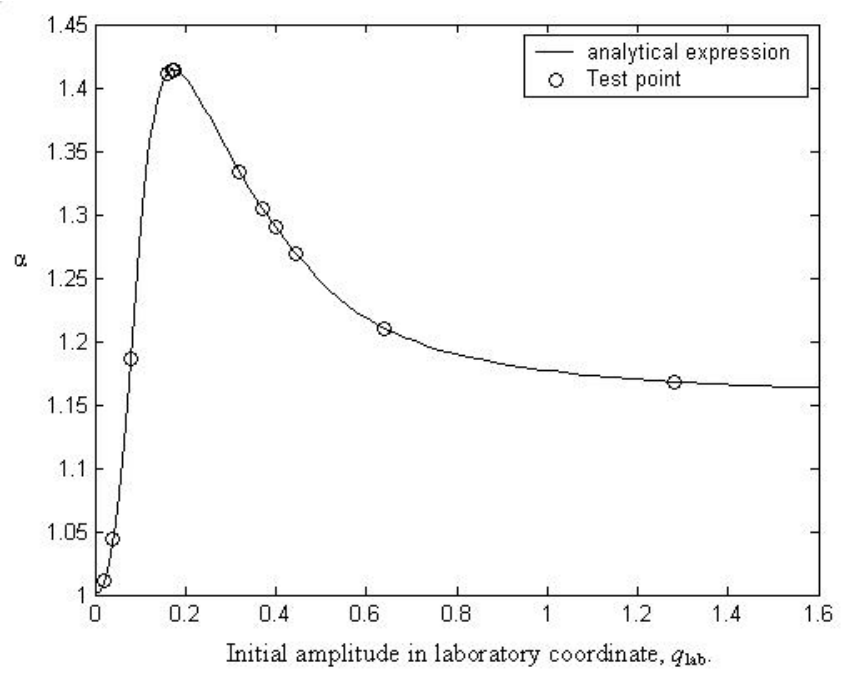

Figure 1 Analytical representation of the maximum amplification factor $\alpha$ (full line) and samples of test points (circles) for various initial amplitudes taking fixed frequencies $\omega_{1_{\mathrm{lab}}}=3.30$ and $\omega_{2_{\mathrm{lab}}}=2.99$.

In Figure 1, we illustrate the graphical representation for the maximum amplification factor for fixed frequencies $\omega_{1_{\mathrm{lab}}}=3.30, \omega_{2_{\mathrm{lab}}}=2.99$ and varying the amplitude. We plot the analytical expressions in full lines while test points are plotted in circles. These points are obtained from tracing the level curve of the Hamiltonian system, substituting the low dimensional mode solution (10), and looking for the point that gives the maximal amplitude. As expected, both ways lead to the same results. We also observed that the least upper bound for the maximum amplification factor is $\sqrt{2}$.

We further compare the results obtained from the low-dimensional model with numerical results obtained from a numerical scheme for the NLS equation. The numerical scheme is based on discretizing the variational structure in the space variable, and using an implicit Crank-Nicolson method for discretizing the time variable. For fixed frequencies $\omega_{1_{\mathrm{lab}}}=3.30, \omega_{2_{\mathrm{lab}}}=2.99$ the results are given in Figure 2. We note that as the initial amplitude $\mathrm{q}_{\mathrm{lab}}$ increases, the maximal 


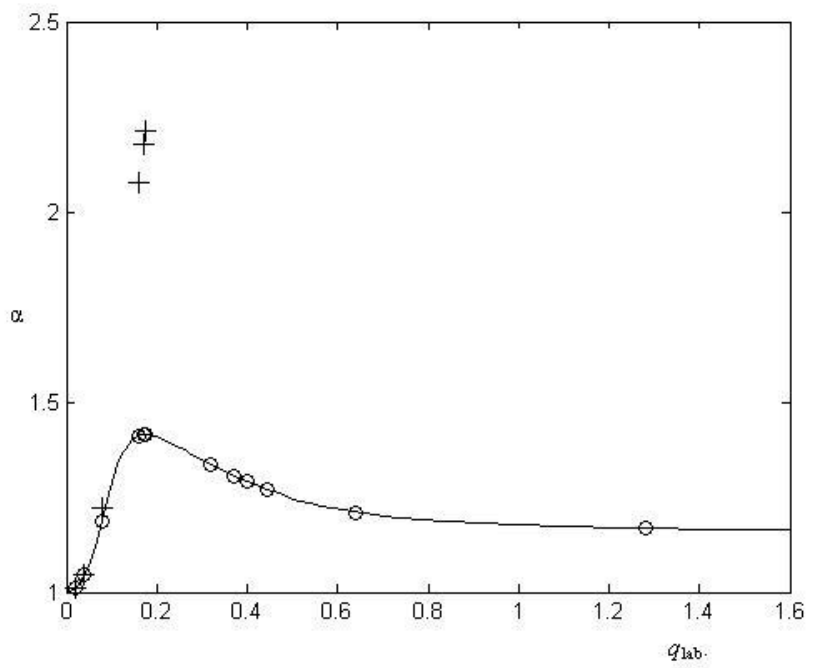

Figure 2 Comparison between the analytical expression (full line), sample test points (circles), and numerical results $(+)$ of the maximum amplification factor $\alpha$ vs amplitude for fixed frequencies $\omega_{1_{\mathrm{lab}}}=3.30$, and $\omega_{2_{\mathrm{lab}}}=2.99$.

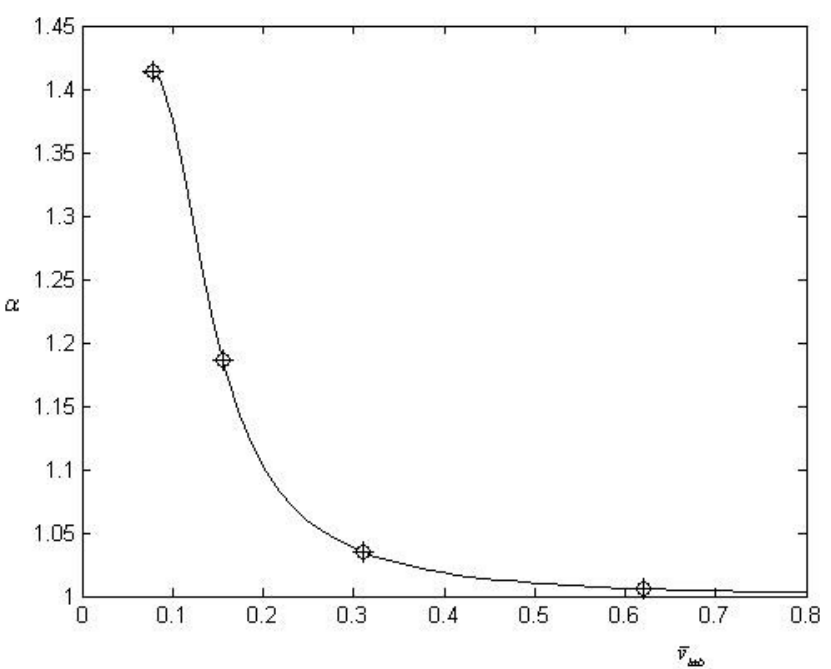

Figure 3 Comparison between the analytical expression (full line), sample test points (circles), and numerical results $(+)$ of the maximum amplification factor $\alpha$ vs $v_{\text {lab }}$ for fixed amplitude $\mathrm{q}_{\text {lab }}=0.08$. 
amplification factor $\alpha$ increases as well, until it reaches its maximal at $\sqrt{2}$ when $q_{l a b}=\frac{-8 \kappa}{\gamma} \sqrt{\gamma \beta}$, after which $\alpha$ decreases and approaches the value $\frac{2 \sqrt{3}}{3}$. The analytical results agree well with the numerical simulations for the range when the initial amplitude is not too large. For fixed amplitude, it is observed that the amplification factor $\alpha$ increases as the difference of frequencies decreases. This is illustrated in Figure 3 for some range of parameter $\bar{v}_{l a b}$ for which the analytical and numerical results are comparable. In the case that $\mathrm{q}_{\text {lab }}$ is reasonably large or $\bar{v}_{\text {lab }}$ is reasonably small, we observe that the analytical results deviate quite significantly with the numerical simulations, see Table 1 and 2 .

\begin{tabular}{|c|c|c|c|}
\hline $\mathrm{q}_{\text {lab }}$ & $a_{0}$ & $\alpha_{\text {low-dim }}$ & $\alpha_{\text {simulation }}$ \\
\hline 0.02 & 0.004 & 1.010669 & 1.010738 \\
\hline 0.04 & 0.008 & 1.043939 & 1.045113 \\
\hline 0.08 & 0.016 & 1.186599 & 1.222400 \\
\hline 0.16 & 0.032 & 1.411478 & 2.075361 \\
\hline 0.174087 & 0.034817 & $\sqrt{2}$ & 2.213500 \\
\hline
\end{tabular}

Table 1 Comparison results of the maximum amplification factor $\alpha$ for various $\mathrm{q}_{\mathrm{lab}}$ taking fixed frequencies $\omega_{\mathrm{lab}_{\mathrm{ab}}}=3.30$, and $\omega_{2_{\mathrm{ab}}}=2.99 . \alpha_{\text {low-dim }}$ is $\alpha$ obtained from the analytical expression while $\alpha_{\text {simulation }}$ is $\alpha$ obtained from the numerical results.

\begin{tabular}{|c|c|c|c|}
\hline $\bar{v}_{\text {lab }}$ & $v$ & $\alpha_{\text {low-dim }}$ & $\alpha_{\text {simulation }}$ \\
\hline 0.62 & 0.442857 & 1.005767 & 1.005767 \\
\hline 0.31 & 0.221429 & 1.035044 & 1.035044 \\
\hline 0.155 & 0.110714 & 1.186599 & 1.222400 \\
\hline 0.0775 & 0.055357 & 1.413899 & 2.16900 \\
\hline
\end{tabular}

Table 2 Comparison of results of the maximum amplification factor $\alpha$ for various $\bar{v}_{\text {lab }}$ taking $\mathrm{q}_{\text {lab }}=0.08$ with $\omega_{\mathrm{l}_{\mathrm{lab}}}=3.30 . \alpha_{\text {low-dim }}$ is $\alpha$ obtained from the analytical expression while $\alpha_{\text {simulation }}$ is $\alpha$ obtained from the numerical results.

It is further noted that the Hamiltonian contour for the dynamics of (5) is a closed orbit as shown in Figure 4. Hence, there is a periodic evolution of $A(\xi, \tau)$ in the low-dimensional model. In our investigation, we start with the first mode. During the evolutions, part of the energy is transferred from the first 
mode to create the other mode. When the phase different is 0 again, the energy of the other mode is transferred back to the first mode until the other mode disappears. Hence, the energy transfer between the first and the other mode is periodic. The energy exchange is larger for larger initial amplitude; for example for the case that $\mathrm{q}_{\text {lab }}=0.02, \mathrm{r}_{1} \in[0.0039997699,0.004]$ and for $\mathrm{q}_{\text {lab }}=0.16$, $r_{1} \in[0.023990381,0.032]$. When the initial amplitude is small, the energy exchange is small, and thus the deformation is smaller. When the energy exchange is larger, there is more energy lost in the first mode. The loss from the first mode is fully transferred to the other mode under the assumptions of this low-dimensional model. However, comparisons with numerical simulations show that the first and other mode fail to capture the actual evolutions when the initial amplitude is large. This indicates that the energy loss from the first-mode is not only transferred to the other mode, but instead will create also higher order modes which will play a vital role in the deformation of the envelope.

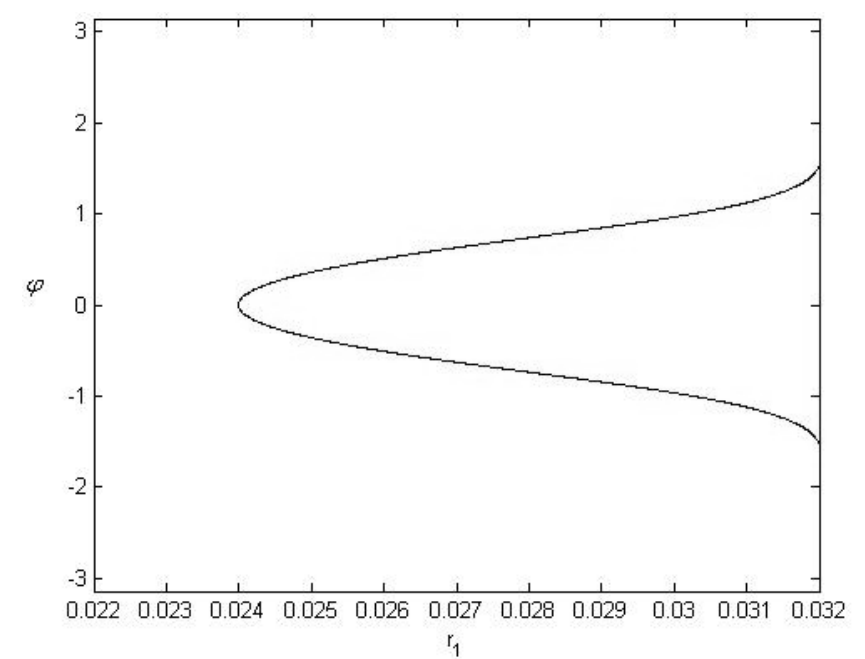

Figure 4 The curve of the constant Hamiltonian $\mathcal{H}^{*}\left(r_{1}, \varphi\right)$ for $\mathrm{q}_{\text {lab }}=0.16$.

\section{Conclusions}

We have considered a low dimensional model of NLS equation for the evolution of surface gravity waves. We have taken as simple case the evolution of bichromatic wave groups with this low dimensional model and looked for the least upper bound for the amplification factor $A F$. This $A F$ is defined as the ratio between the maximum of the bichromatic envelope ever achieved during the evolution and the maximum of the initial envelope over the spatial domain. 
It is analytically found that the least upper bound for $A F$ using this model is $\sqrt{2}$.

Comparisons with numerical solutions of the NLS are made. We found that the solutions of the low-dimensional model are in a good agreement with the numerical solutions of the NLS equation for certain range of parameters, at least when the initial amplitude is not too large and when the difference of frequency is not too small. Out of this range, there is a significant deviation between the low-dimensional and numerical results, which shows that effects from higher order terms increase and dominate the evolutions so that the lower modes can no longer describe the evolutions accurately. Future research will investigate the effects of taking more modes in the evolution or taking higher order models than the low dimensional model used here.

\section{Acknowledgement}

The authors are very thankful to Prof. Brenny van Groesen for many valuable suggestions and discussions. This research is conducted partly at University Teknology Malaysia \& Institut Teknologi Bandung, Indonesia and partly at the University of Twente, The Netherlands. The second author is funded by Riset Unggulan Terpadu Internasional (RUTI 2002/2005) in a collaboration with University of Twente, STW project TWI5734 and Scientific Programme Indonesia Netherlands, Extended Programme in Applied Mathematics.

\section{References}

1. Lo, E. \& Mei, C. C., A numerical study of water-wave modulation based on a higher-order nonlinear Schrödinger equation, J. Fluid Mech., 150, 395 (1985).

2. Stansberg, G., On the nonlinear behaviour of ocean wave groups, proc. Waves '97, 2, 1227-1241 (1997).

3. Westhuis, J., The numerical simulation of nonlinear waves in a hydrodynamic model test basin, Ph.D. Thesis in University of Twente, the Netherlands (2001).

4. Groesen, E. van, Andonowati \& Soewono, E., Non-linear effects in bichromatic surface waves, Proc. Estonian Acad. Sci., Mathematics and Physics v48, pp. 206-229 (1999).

5. Andonowati \& Groesen, E. van, Optical Pulse Deformation in Second Order Non-linear Media, Journal of Nonlinear Optical Physics \& Materials, v.12, n.2, pp. 221-234 (2003).

6. Lighthill, M. J., Contributions to the theory of waves in nonlinear dispersive systems, J. Inst. Math. Appl., 1, 269 (1965). 
7. Benjamin, T. B., Instability of periodic wave trains in nonlinear dispersive systems, Proc. Roy. Soc., A299, 59 (1967).

8. Karjanto, N., Groesen, E. van. \& Peterson, P., Investigation of the maximal amplitude increase from the Benjamin-Feir instability, Journal of Indonesian Mathematical Society, Vol. 8 No. 4, 39 - 47 (2002).

9. Sulem, C. \& Sulem, P.L., The nonlinear Schrödinger Equation -SelfFocusing and Wave Collapse, volume 139 of Applied Mathematical Sciences, Springer (1999).

10. Onorato, M., Osborne, A. R., Serio, M. \& Bertone, S., Freak waves in random oceanic sea states, Phys. Rev. Lett, 86, 5831 (2001).

11. Groesen, E. Van., Wave groups in uni-directional surface wave model, Journal of Engineering Mathematics, 34, 215-226 (1998). 